\title{
Finding the best variables for direct marketing models
}

\author{
RECEIVED: 6 JUNE 2000 \\ Bruce Ratner, PhD \\ DM STAT-I Consulting, 574 Flanders Drive, North Woodmere, Ny II58I, USA \\ Tel: + I 516 3544; Fax: + I 516 79I 5075; e-mail: br@dmstatl.com
}

\begin{abstract}
Finding the best possible subset of variables to put in a model has been a frustrating exercise. Many methods of variable selection exist, but none of them is perfect. Moreover, they do not create new variables which would enhance the predictive power of the original variables themselves. Furthermore, none use a criterion that addresses the specific needs of direct marketing models. After repeated testing, the author has developed a new methodology that uses genetic modelling to find the variables of significance to direct marketers.
\end{abstract}

\section{BACKGROUND}

The problem of finding the best subset of variables in order to define the best model has been extensively studied. Existing methods - based on theory, search heuristics and rules of thumb each use a unique criterion to build the best model. Selection criteria can be divided into two groups: one based on criteria involving classical hypothesis testing and the other involving residual error sum of squares. ${ }^{1}$ Different criteria typically produce different subsets. The number of variables in common with the different subsets is small, and the sizes of the subsets can vary considerably.

Essentially, the problem of variable selection is to examine certain subsets and select the subset that either maximises or minimises an appropriate criterion. Two subsets are obvious the best single variable and the complete set of variables. The problem lies in selecting an intermediate subset that is better than both of these extremes. Therefore, the issue is how to find the necessary variables among the complete set of variables by deleting both irrelevant variables (variables not affecting the dependent variable) and redundant variables (variables not adding anything to the dependent variable). ${ }^{2}$

Reviewed below are five variable selection methods, which are widely used. The first four are found in major statistical software packages ${ }^{3}$ and the last is the favoured rule-of-thumb approach used by many analysts. The test-statistic for the first three methods uses either the $F$ statistic for a continuous dependent variable, or the $G$ statistic for a binary dependent variable (eg response, which assumes only two values, yes/no). The test-statistic for the fourth method is either $R$-squared

\section{Bruce Ratner}

$\mathrm{PhD}$ is President of DM STAT-1 CONSULTING, the leading firm for analysis and modelling in the DM industry, specialising in statistical methods and knowledge discovery and data-mining tools, in the areas of banking, insurance, finance, retail, telecommunications,

business-to-

business, and catalogue marketing. Bruce is the author of the DM STAT-1 Newsletter on the Web at www.dmstat1.com. 
for a continuous dependent variable, or the Score statistic for a binary dependent variable.

Forward selection (FS). This method adds variables to the model until no remaining variable (outside the model) can add anything significant to the dependent variable. It begins with no variable in the model. For each variable, the test-statistic (TS), a measure of the variable's contribution to the model, is calculated. The variable with the largest TS value that is greater than a preset value $C$ is added to the model. Then the test-statistic is calculated again for the variables still remaining, and the evaluation process is repeated. Thus, variables are added to the model one by one until no remaining variable produces a $T S$ value that is greater than $C$. Once a variable is in the model, it remains there.

Backward elimination (BE). This method deletes variables one by one from the model until all remaining variables contribute something significant to the dependent variable. It begins with a model which includes all variables. Variables are then deleted from the model one by one until all the variables remaining in the model have TS values greater than $C$. At each step, the variable showing the smallest contribution to the model (ie with the smallest TS value that is less than $C$ ) is deleted.

Stepwise (SW). This method is a modification of the forward selection approach and differs in that variables already in the model do not necessarily stay. As in forward selection, $S W$ adds variables to the model one at a time. Variables that have a TS value greater than $C$ are added to the model. After a variable is added, however, $S W$ looks at all the variables already included to delete any variable that does not have a TS value greater $C$.

$\mathrm{R}$-squared ( $R$-sq). This method finds several subsets of different sizes that best predict the dependent variable. $R$-sq $(k)$ finds subsets of variables that best predict the dependent variable based on the appropriate TS. The best subset of size $k$ has the largest TS value. For a continuous dependent variable, $T S$ is the popular measure $R$-squared, the coefficient of multiple determination, which measures the proportion of the 'explained' variance in the dependent variable by the multiple regression. For a binary dependent variable, TS is the theoretically correct but lessknown Score statistic. ${ }^{4}$

$R$-sq finds the best one-variable model, the best two-variable model, and so forth. It is unlikely, however, that one subset will stand out as clearly being the best, as TS values are often bunched together. For example, they are equal in value when rounded at the, say, third place after the decimal point. ${ }^{5} R$-sq generates a number of subsets of each size, which allows the user to select a subset, possibly using non-statistical conditions.

Rule-of-thumb-top k variables (Top- $k$ ). This method selects the top ranked variables in terms of their association with the dependent variable. Each variable's association with the dependent variable is measured by the Pearson 
correlation coefficient $r$. The variables are ranked by their absolute $r$ values, ${ }^{6}$ from largest to smallest. The top- $k$ ranked variables are considered to be the best subset. If the regression model with the top- $k$ variables indicates that all variables have $T S$ values greater than $C$, then the set of $k$ variables is declared the best subset. If any variable has a $T S$ value less than $C$, then the variable is removed and replaced by the next ranked variable. The new set of variables is then considered to be the best subset, and the evaluation process is repeated.

\section{WEAKNESSES IN THE VARIABLE SELECTION METHODS}

While these methods produce reasonably good models, each method has a drawback specific to its selection criterion. A detailed discussion of the weaknesses is beyond the scope of this paper, ${ }^{7,8}$ however, there are two common weaknesses that do merit attention.

First, these methods cannot identify structure in the data. They find the best subset of variables without 'digging into the data', a feature which is necessary for finding important variables or structures. Therefore, variable selection methods without 'data mining' capability cannot generate the enhanced best subset.

The following illustration clarifies this weakness. Consider the complete set of variables, $X_{1}, X_{2}, \ldots, X_{10}$. Any of the variable selection methods in current use will only find the best combination of the original variables (say $X_{1}, X_{3}, X_{7}, X_{10}$ ), but can never automatically transform a variable (say transform $X_{1}$ to $\log X_{1}$ ) if it were needed to increase the information content (predictive power) of that variable. Furthermore, none of these methods can generate a re-expression of the original variables (perhaps $X_{3} / X_{7}$ ) if the constructed variable were to offer more predictive power than the original component variables combined. In other words, current variable selection methods cannot find the enhanced best subset, which needs to include transformed and re-expressed variables (possibly $X_{1}, \quad X_{3}, \quad X_{7}, \quad X_{10}, \quad \log X$, $X_{3} / X_{7}$ ). A subset of variables without the potential of new variables offering more predictive power clearly limits the analyst in building the best model.

Specifically, these methods fail to identify structure of the types discussed below.

Transformed variables with a preferred shape. A variable selection procedure should have the ability to transform an individual variable, if necessary, to induce a symmetric distribution. Symmetry is the preferred shape of an individual variable. Symmetry facilitates the interpretation of the variable's effect in an analysis. Skewed distributions are difficult to examine because most of the observations are bunched together at one end of the distribution. A variable selection method should also have the ability to straighten non-linear relationships. A linear or straightline relationship is the 
preferred shape when considering two variables. A straightline relationship between independent and dependent variables is an assumption of the popular linear model. A linear model is defined as a weighted sum of variables, such as $\left.Y=b_{0}+b_{1} \star X_{1}+b_{2} \star X_{2}+b_{3} \star X_{3}.\right)^{9}$ Moreover, straight-line relationships among all the independent variables is a desirable property. ${ }^{10}$ Straightline relationships are easy to interpret: a unit of increase in one variable produces an expected constant increase in a second variable.

Constructed variables from the original variables using simple arithmetic functions. A variable selection method should have the ability to construct simple re-expressions of the original variables. Sum, difference, ratio or product variables potentially offer more information than the original variables themselves.

Constructed variables from the original variables using a set of functions (eg, arithmetic, trigonometric and/or Boolean). A variable selection method should have the ability to construct complex re-expressions with mathematical functions that capture the complex relationships in the data, and potentially offer more information than the original variables themselves.

Secondly, these methods' selection criteria do not explicitly address the specific needs of direct marketing models, namely to maximise the cum lift.

In sum, the two above-mentioned weaknesses suggest that a high-perfor- mance variable selection method for direct marketing models should find the best subset of variables that maximises the cum lift criterion.

\section{GOALS OF MODELLING IN DIRECT MARKETING}

Direct marketers typically attempt to improve the effectiveness of their solicitations by targeting their best customers or prospects. They use a model to identify individuals who are likely to respond to or generate profit $^{11}$ from a solicitation. The model provides, for each individual, estimates of probability of response and estimates of contribution-to-profit. Although the precision of these estimates is important, the model's performance is measured at an aggregated level as reported in a decile analysis.

Direct marketers have defined the cum lift, which is found in the decile analysis, as the relevant measure of model performance. Based on the model's selection of individuals, direct marketers create a solicitation list to obtain an advantage over a random selection of individuals. The cum response lift is an index of how many more responses are expected with a selection based on a model over the responses expected with a random selection (without a model). Similarly, the cum profit lift is an index of how much more profit is expected with a selection based on a model over the profit expected with a random selection (without a model). The concept of cum lift is best understood by going 
through the steps in the construction of a decile analysis.

\section{DECILE ANALYSIS}

The decile analysis is a tabular display of model performance. Construction of the response decile analysis consists of the following steps (see Table 1):

1 score the sample or file using the model under consideration. Every individual receives a model score, Prob_est, the model's estimated probability of response

2 rank the scored file, in descending order by Prob_est

3 divide the ranked and scored file into ten equal groups. The decile variable is created, which takes on ten ordered 'values': top (1), 2, 3, 4, 5, 6, 7, 8, 9 and bottom (10). The 'top' decile consists of the best 10 per cent of individuals most likely to respond; decile 2 consists of the next 10 per cent of individuals most likely to respond. And so on, for the remaining deciles. Accordingly, the deciles separate and order the individuals on an ordinal scale ranging from most likely to least likely to respond

4 number of individuals is the number of individuals in each decile; 10 per cent of the total size of the file

5 number of responses is the actual - not predicted - number of responses in each decile. The model identifies 911 actual respondents in the top decile. In decile 2, the model identifies 544 actual respon- dents. And so on, for the remaining deciles

6 decile response rate is the actual response rate for each decile group. It is number of responses divided by number of individuals for each decile group. For the top decile, the figures are 12.3 per cent $(=911 / 7,410)$. For the second decile, they are 7.3 per cent $(=544 / 7,410)$. Similarly for the remaining deciles

7 cumulative response rate for a given depth-of-file (the aggregated or cumulative deciles) is the response rate among the individuals in the cumulative deciles. For example, the cumulative response rate for the top decile (10 per cent depth-of-file) is 12.3 per cent $(=911 / 7,410)$. For the top two deciles $(20$ per cent depth-of-file), the cumulative response rate is 9.8 per cent $=([911+544] /[7410+7410])$. Similarly for the remaining deciles

8 cum response lift - for a given depth-of-file — is the cumulative response rate divided by the overall response rate of the file, multiplied by 100 . It measures how much better one can expect to do with the model than without the model. For example, a cum lift of 294 for the top decile means that when soliciting to the top 10 per cent of the file based on the model, 2.94 times the total number of respondents found by soliciting 10 per cent of file can be expected. The cum lift of 235 for the top two deciles, means that when soliciting to 20 per cent of 
the file based on the model, the marketer can expect 2.35 times the total number of respondents found by soliciting 20 per cent of file without any model. Similarly for the remaining deciles.

The calculation for a profit decile analysis along with the cum profit lift follows the steps above with one modification. The response variable (the number of responses) is replaced by the profit variable (the total profit).

It should be clear at this point that a model that produces a decile analysis with more responses or total profit in the upper (top, 2, 3 or 4) deciles is a better model than a model with fewer responses or less total profit in the upper deciles. This concept is the motivation for the GMAX model.

\section{THE GMAX MODEL}

The GMAX approach to modelling is to address specifically the objectives concerning direct marketers, namely, maximising response and profit from solicitations. The GMAX model uses the genetic methodology explicitly to optimise the desired criterion: maximising the upper deciles. Consequently, the GMAX model allows data analysts to build response and profit models in ways that are not possible with current methods.

The GMAX response and profit models are theoretically superior with respect to maximising the upper deciles - to response and profit models built with alternative techniques because of the explicit nature of the fitness function. The actual formulation of the fitness function is beyond the scope of this paper; but suffice to say, the fitness function seeks to fill the upper deciles with as many responses or as much profit as possible. For more about the GMAX models see Bruce Ratner's article 'Genetic modelling in direct marketing' on the web at http://www.dmstat1.com/gene.html.

Due to the explicit nature of its fitness criterion and the way it evolves models, the GMAX model offers high-performance variable selection for direct marketing models. This will become apparent once the GMAX modelling process is illustrated in the next section.

\section{WHAT IS GENETIC MODELLING?}

Just as Darwin's principle of the survival of the fittest explains tendencies in human biology, direct marketers can use the same principle to predict the best current solution to an optimisation problem. ${ }^{12}$ Each genetic model has an associated fitness value that indicates how well the model solves, or 'fits' the problem. A model with a high fitness value solves the problem better than a model with a lower fitness value, and survives and reproduces at a high rate. Models that are less fit survive and reproduce, if at all, at a lower rate.

If two models are effective in solving a problem then some of their parts undoubtedly contain some valuable genetic material. Recombining the 
parts of highly fit 'parent' models can sometimes produce 'offspring' models that are better fit at solving the problem than either parent. Offspring models then become the parents of the next generation, repeating the recombination process. After many generations, an evolved model is declared the best-so-far solution of the problem.

Genetic modelling ${ }^{13,14}$ consists of the following steps:

1 definition of the fitness function. The fitness function should describe what is the best model, and allow for identifying and modifying good or bad models

2 selection of a set of functions (eg the set of arithmetic operators addition, subtraction, multiplication, division $\}$; $\log$ and exponential) and variables (predictors $X_{1}, X_{2}, \ldots, X_{n}$, and numerical constants) believed to be related to the problem at hand (the dependent variable $Y$ ). ${ }^{15}$ An initial population of random models must be generated, using the preselected set of functions and variables

3 calculation of the fitness of each model in the population by applying the model to a training set, a sample of individuals along with their values on the predictor variables, $X_{1}, X_{2}$, $\ldots, X_{n}$, and the dependent variable $Y$. Thus, every model has a fitness value reflecting how well it solves the problem

4 creation of a new population of models by applying the following three operations. The operations are applied to models in the current population selected with a probability based on fitness (ie the fitter the model, the more likely the model is to be selected): (a) reproduction: copying an existing model to the new population; (b) crossover: creation of two offspring models for the new population by genetically recombining randomly chosen parts of two existing parent models; (c) mutation: introduction of random changes in some models

5 the model with the highest fitness value produced in a generation is declared the best-of-generation model, which may be the solution, or an approximate solution to the problem.

\section{GENETIC MODELLING: AN ILLUSTRATION}

This section looks at the process of building a response model. The best model is designated as one with the highest $R$-squared value. ${ }^{16}$ Thus, the fitness function is the formula for $R$ squared.

The modeller has to decide on functions and variables that are related to the problem at hand (eg predicting response). Unless there is theoretical justification, empirical experience, usually based on trial and error, provides the guidance for the functions and variables.

There are two variables, $X_{1}$ and $X_{2}$, to use as predictors of response. Thus, the variable set contains $X_{1}$ and $X_{2}$. 
The numerical constant ' $b$ ' is added to the variable set based on prior experience. The function set is defined to contain the four arithmetic operations and the exponential function (exp), also based on prior experience.

Generating the initial population is done with an unbiased function roulette wheel, and an unbiased function-variable roulette wheel. The slices of the function wheel are of equal size, namely, 20 per cent. The slices of the function-variable wheel are of equal size, namely, 12.5 per cent. (See Figures 1 and 2.) Note, the division symbol ' $\%$ ' is used to denote a 'protected' division. This means that division by zero is set to the value 1 .

To generate the first random model, the function wheel is spun. The wheel's pointer lands on slice ' + '. Next, the function-variable wheel is spun; the pointer lands on slice $X_{1}$. From the next two spins of the function-variable wheel, the pointer lands on slices ' + ' and ' $b$ ', successively. One can decide to stop evolving the model at this point. The resultant random model 1 is depicted as a rooted point-label tree. (See Figure 3.)

The second random model is generated by spinning the function wheel once, then by spinning the functionvariable wheel twice. The pointer lands on slices ',$+ \quad X_{1}$ and $X_{1}$, successively. Model 2 is in Figure 4. Similarly, three more random models, 3, 4 and 5, are generated. (See Figures 5, 6 and 7.) Thus, the initial population of five random models (population size is five) has been generated.
Each of the five models in the population is assigned a fitness value indicating how well it solves the problem of predicting response. Because $R$-squared is being used as the fitness function, each model is applied to a training dataset and its $R$-squared value calculated. Model 1 produces the highest $R$-squared value, 0.52 , and model 5 produces the lowest $R$ squared value, 0.05. (See Table 2.)

Fitness for the population itself can be calculated. The total fitness of the population is the sum of the fitness values among all (five) models in the population. Here, the population total fitness is 1.53. (See Table 1.)

\section{Reproduction}

After the initial population is generated, the operation of reproduction takes place. Reproduction is the process by which models are duplicated or copied based on selection proportional to fitness (PTF). PTF is defined as: model fitness value divided by population total fitness (see Table 1). For example, model 1 has a PTF value of $0.34(=0.52 / 1.53)$.

Reproduction PTF means that a model with a high PTF value has a high probability of being selected for inclusion in the next generation. The reproduction operator is implemented with a biased model roulette wheel, where the slices are sized according to PTF values. (See Figure 8.)

The operation of reproduction proceeds as follows. The spin of the biased model roulette wheel determines which models are copied and 
Figure 1: Unbiased function roulette wheel

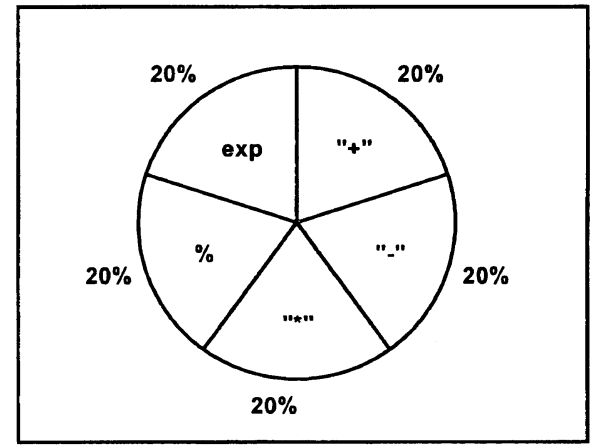

Figure 3: Random model 1

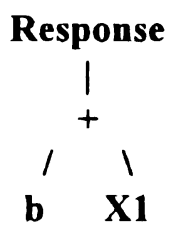

Model 1: Response $=\mathbf{b}+\mathbf{X 1}$

Figure 5: Random model 3

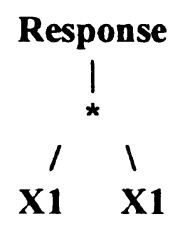

Model 3: Response $=\mathbf{X} 1 * \mathbf{X} 1$
Figure 2: Unbiased function-variable roulette wheel

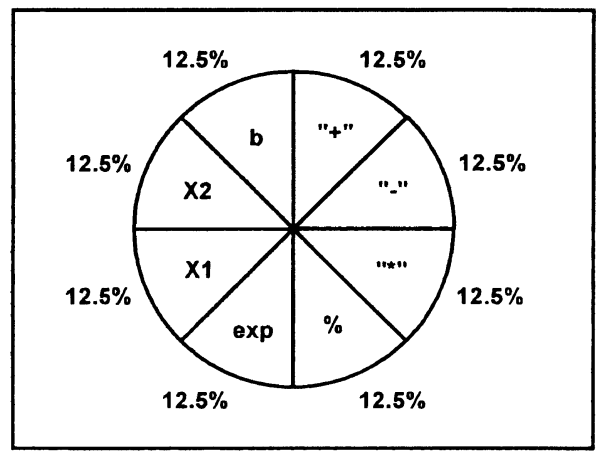

Figure 4: Random model 2

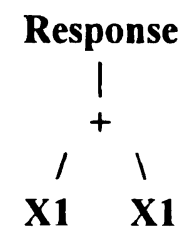

Model 2: Response $=\mathrm{X} 1+\mathrm{X} 1$

Figure 6: Random model 4

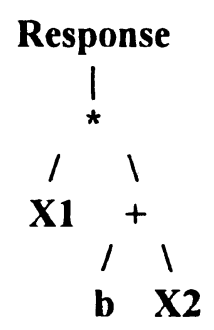

Model 4: Response $=X 1$ * $(b+X 2)$ 
Figure 7: Random model 5

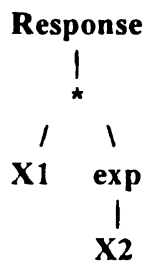

Model 5: Response $=\mathrm{X} 1{ }^{*} \exp (\mathrm{X} 2)$

the number of copies. The model selected by the pointer is copied, without alteration, and put into the next generation. Spinning the wheel in Figure 8, say, 100 times produces on average the selection: 34 copies of model 1, 28 copies of model 2, 25 copies of model 3, 11 copies of model 4 , and 3 copies of model 5 .

\section{Crossover}

The crossover (sexual recombination) operation is performed on two parent models by genetically recombining randomly chosen parts of the two existing parent models, the expectation being that the offspring models are even more fit than either parent model.

The crossover operation works with selection PTF. An illustration makes this operation easy to understand. Consider the two parent models in

Table 1

\section{RESPONSE DECILE ANALYSIS}

\begin{tabular}{llllll}
\hline Decile & $\begin{array}{l}\text { Number of } \\
\text { individuals }\end{array}$ & $\begin{array}{l}\text { Number of } \\
\text { responses }\end{array}$ & $\begin{array}{l}\text { Decile } \\
\text { response } \\
\text { rate } \%\end{array}$ & $\begin{array}{l}\text { Cumulative } \\
\text { response } \\
\text { rate } \%\end{array}$ & Cum lift \\
top & 7,410 & 911 & 12.3 & 12.3 & 294 \\
2 & 7,410 & 544 & 7.3 & 9.8 & 235 \\
3 & 7,410 & 437 & 5.9 & 8.5 & 203 \\
4 & 7,410 & 322 & 4.3 & 7.5 & 178 \\
5 & 7,410 & 258 & 3.5 & 6.7 & 159 \\
6 & 7,410 & 188 & 2.5 & 6.0 & 143 \\
7 & 7,410 & 130 & 1.8 & 5.4 & 129 \\
8 & 7,410 & 163 & 2.2 & 5.0 & 119 \\
9 & 7,410 & 124 & 1.7 & 4.6 & 110 \\
bottom & 7,410 & 24 & 0.3 & 4.2 & 100 \\
Total & 74,100 & 3,101 & 4.2 & &
\end{tabular}


Table 2

\section{INITIAL POPULATION}

$\begin{array}{ll}\text { Fitness value } & \text { PTF } \\ (R \text {-squared }) & \text { (fitness/total) }\end{array}$

$\begin{array}{lll}\text { Model } 1 & 0.52 & 0.34 \\ \text { Model } 2 & 0.41 & 0.28 \\ \text { Model } 3 & 0.38 & 0.25 \\ \text { Model } 4 & 0.17 & 0.11 \\ \text { Model } 5 & 0.05 & 0.03 \\ \text { Population total } & 1.53 & 1\end{array}$

fitness (PTF)

Figure 8: Biased model roulette wheel



Figures 9 and 10. The operation begins by randomly selecting an internal point (a function) in the tree for the crossover site. Say, the crossover sites are the lower ' + ' and ' $\star$ ' for parents 1 and 2 , respectively.
The crossover fragment for a parent is the subtree that has at its root the crossover site function. Crossover fragments for parents 1 and 2 are in Figures 11 and 12, respectively. Offspring 1 from parent 1 is produced by 
Figure 9: Parent 1

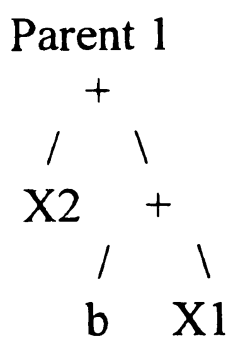

Figure 10: Parent 2

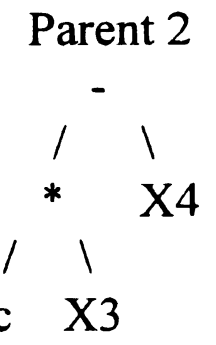

Figure 11: Crossover fragment 1

\section{Crossover Fragment 1}

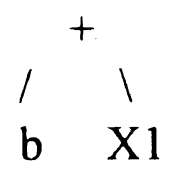

deleting the crossover fragment of parent 1 and then inserting the crossover fragment of parent 2 at the crossover point of the parent 1. (See Figures 9, 12 and 13.) Similarly, offspring 2 is produced. (See Figures 10, 11 and 14.)

\section{Mutation}

The mutation operation begins by selecting a point at random within a tree. This mutation point can be an internal point (a function) or an external or terminal point (a variable or
Figure 12: Crossover fragment 2

\section{Crossover Fragment 2}

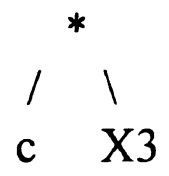

numerical constant). The mutation operation either replaces a randomly generated function with another function (from the function set previously defined), or inverts ${ }^{17}$ the terminals of the subtree whose root is the randomly selected internal point.

For example, model $\mathrm{I}$ is mutated by replacing the function '- ' with ' + ', resulting in mutated model I.1. (See Figures 15 and 16.) Model $\mathrm{I}$ is also mutated by inverting the terminal points, $c$ and $X_{3}$, resulting in mutated Model I.2. (See Figure 17.) 
Figure 13: Offspring 1

\section{Offspring 1}

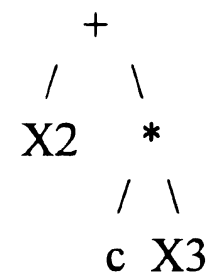

Figure 15: Model I for mutation.

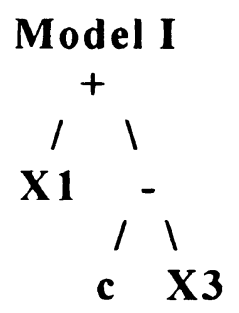

Figure 17: Mutated model I.2

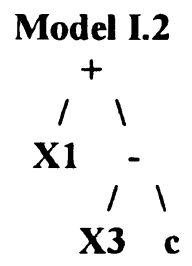

\section{PARAMETERS FOR CONTROLLING A GENETIC MODEL RUN}

There are several control parameters that need to be set before evolving a genetic model:
Figure 14: Offspring 2

\section{Offspring 2}

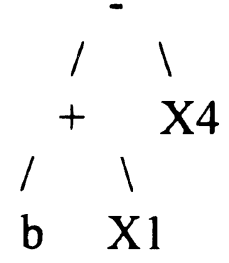

Figure 16: Mutated model I.1



- population size, or the number of models randomly generated, and subsequently evolved

- the maximum number of generations to be run

- reproduction probability or the percentage of the population which is copied. If population size is 100 and reproduction probability is 10 per cent, then ten models from each generation are selected (with reselection allowed) for reproduction. Selection is based on PTF

- crossover probability or the percentage of the population which is 
used for crossover. If population size is 100 and crossover probability is 80 per cent, then 80 models from each generation are selected (with reselection allowed) for crossover. Selection is based on PTF. Models are paired at random

- mutation probability, or the percentage of the population which is used for mutation. If population size is 100 and mutation 10 per cent, then ten models from each generation are selected (with reselection allowed) for mutation. Selection is based on PTF.

- termination criterion, or the single model with the largest fitness value over all generations, the so-called best-so-far model, is declared the result of a run.

\section{GENETIC MODELLING: STRENGTHS AND LIMITATIONS}

Genetic modelling has strengths and limitations, like any methodology. Perhaps the most important strength of genetic modelling is that it is a workable alternative to traditional methods, which are highly parametric with sample size restrictions. Traditional methods have algorithms depending on smooth, unconstrained functions with the existence of derivatives (welldefined slope values). In practice, the functions (response surfaces) are noisy, multimodal and frequently discontinuous. In contrast, genetic models are robust, assumption free, nonparametric and perform well on large and small samples. The only requirement is a fitness function, which can be designed to ensure that the genetic model does not perform worse than any other method.

Genetic modelling has shown itself to be effective for solving large optimisation problems, and searching very large data sets. In addition, genetic modelling can be used to learn complex relationships, making it a viable data mining tool for digging among the data to find 'golden nuggets' of information.

A potential limitation of genetic modelling is in the setting of the genetic modelling parameters: population size, reproduction, crossover and mutation probabilities. The parameter settings are, in part, data and problem dependent, thus proper settings require experimentation. Fortunately, new theories and empirical studies are continually providing rules of thumb for these settings as application areas broaden. These guidelines make genetic modelling an accessible approach to analysts not formally trained in genetic modelling. Even with the 'correct' parameter settings, genetic models do not guarantee the optimal (best) solution. Further, genetic models are only as good as the definition of the fitness function. Precisely defining the fitness function, sometimes requires expert experimentation.

\section{VARIABLE SELECTION WITH GMAX}

The best way of explaining variable selection with the GMAX model is to 
Table 3

\section{CORRELATION ANALYSIS AMONG THE NINE ORIGINAL VARIABLES - PEARSON CORRELATION COEFFICIENT WITH RESPONSE \\ (variable/correlation coefficient $\mathrm{r} / \mathrm{p}$-value)}

\begin{tabular}{lllll}
\hline DOLLAR_2 & RFM_CELL & PROD_TYP & LSTORD_M & AGE_Y \\
0.11202 & -0.10413 & 0.07900 & -0.07604 & 0.03577 \\
6.E-44 & 3.E-38 & $1 . E-22$ & $4 . E-21$ & $9 . E-06$ \\
& & & & \\
PROMOTIO & AVG_ORDE & OWN_TEL & FSTORD_M & \\
0.03409 & 0.02259 & 0.00977 & 0.00901 & \\
2.E-05 & 0.00517 & 0.22653 & 0.26490 & \\
\hline
\end{tabular}

illustrate how GMAX identifies structure in data. In this illustration, the structure for a response model is found. The same holds true, however, for a profit model with a continuous dependent variable.

Cataloguer $\mathrm{ABC}$ requires a response model to be built on a recent mail campaign that produced a 3.54 per cent response rate. In addition to the RESPONSE dependent variable, there are nine candidate predictor variables, whose measurements were taken prior to the mail campaign:

- AGE_Y: knowledge of customer's age $(1=$ if known; $0=$ if not known)

- OWN_TEL: presence of telephone in the household $(1=$ yes; $0=$ no)

- AVG_ORDE: average dollar order

- DOLLAR_2: dollars spent within last two years
- PROD_TYP: number of different products purchased

- LSTORD_M: number of months since last order

- FSTORD_M: number of months since first order

- RFM_CELL: recency/frequency/ money cells $(1=$ best to $5=$ worse $^{18}$

- PROMOTIO: number of promotions customer has received.

As a preliminary step to gauge the information content (or predictive power) of the variables, a correlation analysis is performed. The Pearson correlation coefficients (Table 3) indicate the strength of association between each variable and RESPONSE. There are seven statistically significant variables at the confidence level of at least 95 per cent ( $p$-values less than/equal to 
Table 4

BEST SUBSETS AMONG THE NINE ORIGINAL VARIABLES

\begin{tabular}{llllll}
\hline Method & DOLLAR_2 & RFM_CELL & LSTORD-M & AGE_Y & AVE_ORDE \\
FS & $\mathrm{x}$ & $\mathrm{x}$ & $\mathrm{x}$ & $\mathrm{x}$ & \\
BE & $\mathrm{x}$ & $\mathrm{x}$ & $\mathrm{x}$ & $\mathrm{x}$ & \\
SW & $\mathrm{x}$ & $\mathrm{x}$ & $\mathrm{x}$ & $\mathrm{x}$ & \\
$R-\mathrm{sq}$ & $\mathrm{x}$ & $\mathrm{x}$ & $\mathrm{x}$ & $\mathrm{x}$ \\
Top-4 & $\mathrm{x}$ & $\mathrm{x}$ & $\mathrm{x}$ & $\mathrm{x}$ \\
\hline
\end{tabular}

0.05). The variables in descending order of the magnitude ${ }^{19}$ of the strength of association are: DOLLAR_2, RFM_CELL, PROD_TYP, LSTORD_M, AGE_Y, PROMOTIO and AVG_ORDE.

Five logistic regression analyses (with RESPONSE) were performed, corresponding to the five variable selection methods. The resulting best subsets among the nine original variables are represented in Table 4. Surprisingly, the forward, backward and stepwise methods produced the identical subset (DOLLAR_2, RFM_CELL, LSTORD_M, AGE_Y). Because these methods produced a subset size of 4 , the subset size was set to 4 for the $R$-sq and Top- $k$ methods. This allows a fair comparison across all methods. $R$-sq and Top- $k$ produced different best subsets, which include DOLLAR_2, LSTORD_M and AVG_ORDE. (See Table 4.)

The performance of the five logistic models in terms of cum lift is reported in Table 5. Assessment of model performance at the decile level is as follows:

- at the top decile, $R$-sq produced the worst performing model: cum lift 239 vs. cum lifts 252-256 for the other models

- at the second decile, $R$-sq produced the worst performing model: cum lift 198 vs. cum lifts 202-204 for the other models

- at the third decile, $R$-sq produced the best performing model: cum lift 178 vs. cum lifts $172-174$ for the other models

- similar findings can be made at the other depths-of-file.

To facilitate the comparison of the five statistics-based variable selection methods and the proposed geneticbased technique, a single measure of model performance is used for the five 
Table 5

MODEL PERFORMANCE COMPARISON BY VARIABLE SELECTION METHODS: CUM LIFT

\begin{tabular}{lllllll}
\hline Decile & FS & BE & SW & $R$-sq & Top-4 & AVG \\
top & 256 & 256 & 256 & 239 & 252 & 252 \\
2 & 204 & 204 & 204 & 198 & 202 & 202 \\
3 & 174 & 174 & 174 & 178 & 172 & 174 \\
4 & 156 & 156 & 156 & 157 & 154 & 156 \\
5 & 144 & 144 & 144 & 145 & 142 & 144 \\
6 & 132 & 132 & 132 & 131 & 130 & 131 \\
7 & 124 & 124 & 124 & 123 & 121 & 123 \\
8 & 115 & 115 & 115 & 114 & 113 & 114 \\
9 & 107 & 107 & 107 & 107 & 107 & 107 \\
bottom & 100 & 100 & 100 & 100 & 100 & 100 \\
\hline
\end{tabular}

methods. The 'average' performance of the five models is measured by AVG, the average of the cum lifts across the five methods for each decile. (See Table 5.)

\section{GMAX modelling}

The parameters for controlling the GMAX model are set to run as follows:

- population size: 3,000 (models)

- number of generations: 250

- percentage of the population copied: 10 per cent

- percentage of the population used for crossover: 80 per cent

- percentage of the population used for mutation: 10 per cent.
The GMAX-variable set consisted of the nine candidate predictor variables. For the GMAX-function set, the arithmetic functions (addition, subtraction, multiplication and division), some Boolean operators (and, or, xor, greater/less than) and the $\log$ function $(\mathrm{Ln})$ were selected. The $\log$ function $^{20}$ is helpful in symmetrising skewed variables, such as dollar amount variables, which typically are skewed. It was anticipated that DOLLAR_2 would be part of a genetically-evolved structure defined with the log function. Of course, RESPONSE is the dependent variable.

At the end of the run, 250 generations of copying/crossover/mutation have evolved $750,000(250 \times 3,000)$ 
models according to fitness-proportionate selection. Each model was evaluated in terms of how well it solves the problem of 'filling the upper deciles with responders'. Good models having more respondents in the upper deciles and are more likely to contribute to the next generation of models; poor models having fewer respondents in the upper deciles are less likely to contribute to the next generation of models. Consequently, the last generation consists of 3,000 high-performance models, each with a fitness value indicating how well the model solves the problem. The 'top' fitness values, typically the 50 largest values, ${ }^{21}$ define a set of 50 'best' models with equivalent performance (filling the upper deciles with an equivalently large number of responders).

The set of variables defining one of the 'best' models has variables in common with the set of variables defining another 'best' model. The 'common' variables can be considered for the best subset. The mean incidence of a variable across the set of best models provides a measure for determining the best subset. The GMAX-selected best subset of original variables consists of variables with mean incidence greater than $0.75 .^{22}$ The variables that meet this cut-off score reflect an honest determination of necessary variables with respect to the criterion of maximising the deciles.

Returning to the illustration, GMAX provides the mean incidence of the nine variables across the set of 50 best models. The
Table 6

MEAN INCIDENCE OF ORIGINAL VARIABLES ACROSS THE SET OF 50 BEST MODELS

\begin{tabular}{ll}
\hline Variable & Mean incidence \\
& \\
DOLLAR_2 & 1.43 \\
RFM_CELL & 1.37 \\
PROD_TYP & 1.22 \\
AGE_Y & 1.11 \\
LSTORD_M & 0.84 \\
& \\
PROMOTIO & 0.67 \\
AVG-ORDE & 0.37 \\
OWN-TEL & 0.11 \\
FSTORD-M & 0.09 \\
\hline
\end{tabular}

GMAX-selected best subset consists of five variables: DOLLAR_2, RFM_CELL, PROD_TYP, AGE_Y and LSTORD_M. This genetic-based best subset has four variables in common with the statistics-based best subsets (DOLLAR_2, RFM_CELL, LSTORD_M, and AGE_Y). Unlike the statistics-based methods, GMAX finds value in PROD_TYP and includes it in the best subset. (See Table 7.)

At this point, the predictive power of the genetic-based and statisticsbased best subsets can be assessed by comparing logistic regression models with each subset. After identifying the GMAX-evolved structure, the marketer can, however, choose to make a more fruitful comparison. 
Table 7

BEST SUBSETS AMONG ORIGINAL VARIABLES: STATISTICS- AND

GENETIC-BASED VARIABLE SELECTION METHODS

Method DOLLAR_2 RFM_CELL LSTORD-M AGE-Y AVG-ORDE PROD_TYP

$\begin{array}{lllllll}\text { FS } & \mathrm{x} & \mathrm{x} & \mathrm{x} & \mathrm{x} & & \\ \text { BE } & \mathrm{x} & \mathrm{x} & \mathrm{x} & \mathrm{x} & & \\ \text { SW } & \mathrm{x} & \mathrm{x} & \mathrm{x} & \mathrm{x} & & \\ R \text {-sq } & \mathrm{x} & & \mathrm{x} & \mathrm{x} & \mathrm{x} & \\ \text { Top-4 } & \mathrm{x} & \mathrm{x} & \mathrm{x} & & \mathrm{x} & \mathrm{x} \\ \text { GMAX } & \mathrm{x} & \mathrm{x} & \mathrm{x} & \mathrm{x} & \end{array}$

\section{GMAX-structure identification}

Just as in nature, where structure is the consequence of natural selection, as well as sexual recombination and mutation, the GMAX model evolves structure via fitness-proportionate selection (natural selection), crossover (sexual recombination) and mutation. GMAX fitness leads to structure, which is evolved with respect to the criterion of maximising the deciles. Important structure is found in the best models, typically, the models with the four largest fitness values.

Continuing with the illustration, GMAX has evolved several structures, or GMAX-constructed variables. The GMAX Model (in Figure 18a) has the largest fitness value and reveals five new variables, NEW_VAR1 NEW_VAR5. Additional structures are found in the remaining three best models: NEW_VAR6 - NEW_VAR8 (in Figure 18b), NEW_VAR9 (in Figure 18c), and NEW_VAR10 (in Figure 18d):

- NEW_VAR1 = DOLLAR_2/ AGE_Y; if Age_Y $=0$ then NEW_VAR $1=1$

- NEW_VAR2 $=(\text { DOLLAR_2 })^{\star}$ NEW_VAR1

- NEW_VAR3 = NEW_VAR2/ LSTORD_M; if LSTORD_M= then NEW_VAR3 $=1$

- NEW_VAR4 $=$ Ln(NEW_VAR3); if NEW_VAR3 not equal to 0 then NEW_VAR $4=1$

- NEW_VAR $5=$ RFM_CELL / PROD_TYP; if PROD_TYP $=0$ then NEW_VAR5 $=1$

- NEW_VAR6 = RFM_CELL/ DOLLAR_2; if DOLLAR_2 $=0$ then NEW_VAR6 $=1$

$-\mathrm{NEW}_{-}$VAR7 $=$PROD_TYP/ NEW_VAR6; if NEW_VAR6 $=0$ then NEW_VAR7 $=1$ 
Figure 18a: GMAX model, best \#1

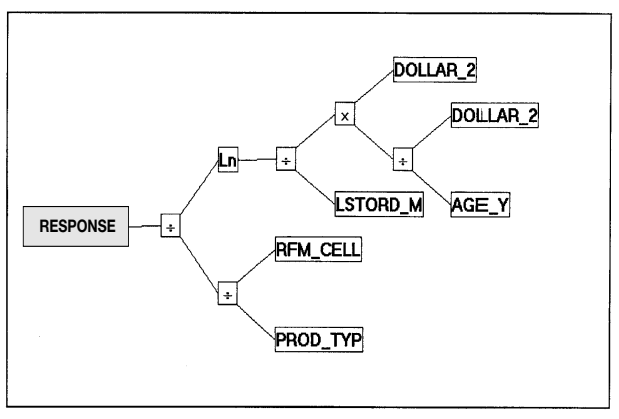

Figure 18b: GMAX model, best \#2

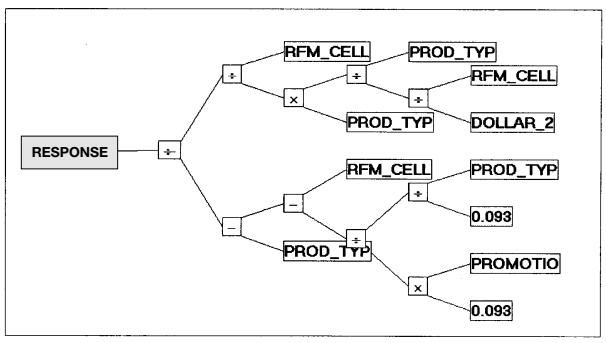

Figure 18c: GMAX model, best \#3

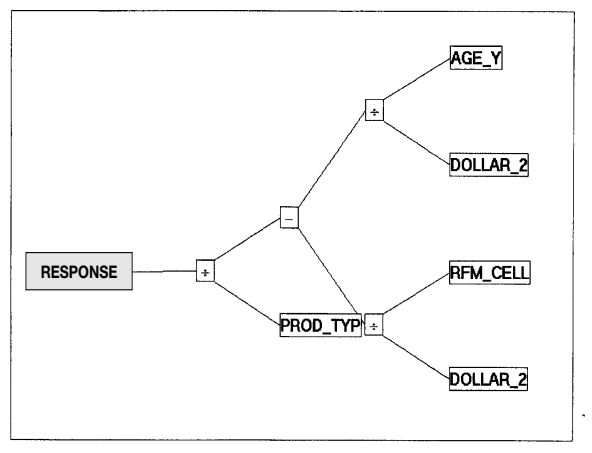

Figure 18d: GMAX model, best \#4

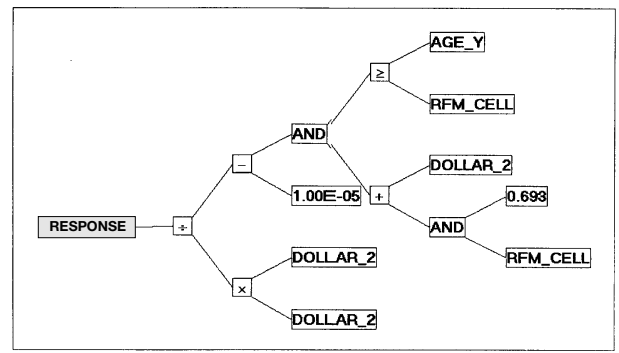


Table 8

\section{CORRELATION ANALYSIS OF THE ORIGINAL AND \\ GMAX-CONSTRUCTED VARIABLES PEARSON CORRELATION \\ COEFFICIENT WITH RESPONSE \\ (variable/correlation coeff $\mathrm{r} / \mathrm{p}$-value)}

\begin{tabular}{lllll}
\hline NEW_VAR7 & NEW_VAR5 & NEW_VAR8 & NEW_VAR1 & DOLLAR_2 \\
0.15592 & 0.15555 & 0.12145 & 0.11905 & 0.11202 \\
3.E-55 & $7 . E-55$ & $2 . E-51$ & $9 . E-45$ & $6 . E-44$ \\
& & & & \\
RFM_CELL & NEW_VAR10 & NEW_VAR4 & PROD_TYP & LSTORD_M \\
-0.10413 & 0.10039 & 0.09773 & 0.07900 & -0.07604 \\
3.E-38 & 1.E-35 & $8 . E-34$ & $1 . E-22$ & $4 . E-21$ \\
& & & & \\
NEW_VAR2 & NEW_VAR3 & NEW_VAR9 & AGE_Y & PROMOTIO \\
0.07326 & 0.06090 & 0.05212 & 0.03577 & 0.03409 \\
1.E-19 & $5 . E-14$ & $1 . E-10$ & $9 . E-06$ & $2 . E-05$ \\
& & & & \\
NEW_VAR6 & AVG_ORDE & OWN_TEL & FSTORD_M & \\
-0.02302 & 0.02259 & 0.00977 & 0.00901 & \\
0.00438 & 0.00517 & 0.22653 & 0.26490 & \\
& & & & \\
\hline
\end{tabular}

- NEW_VAR $8=$ NEW_VAR $7 \star$ PROD_TYP

$-\mathrm{NEW}_{-}$VAR $9=($ A GE_ DOLLAR_2) - (RFM_CELL/ DOLLAR_2); if DOLLAR_2=0 then NEW_VAR9 $=0$

$-\mathrm{NEW}$ _VAR10 $=1$ if AGE_Y greater than/equal RFM_CELL; otherwise $=0$.

To measure the predictive power of the new GMAX-constructed variables, a correlation analysis of the nine original variables and the ten new variables with RESPONSE was per- formed. All the new variables are statistically significant at the 95 per cent or higher confidence level. Some new variables have a stronger association with RESPONSE than the original variables. Specifically, the following associations are observed in Table 8:

- NEW_VAR7, NEW_VAR5, NEW_VAR8 and NEW_VAR1 have a stronger association with RESPONSE than the best original variable DOLLAR_2

- NEW_VAR10 and NEW_VAR4 
Table 9

BEST SUBSETS AMONG THE ENHANCED BEST SUBSET VARIABLES

\begin{tabular}{|c|c|c|c|c|c|c|c|c|}
\hline Method & $\begin{array}{l}\text { DOLLAR_ } \\
2\end{array}$ & $\begin{array}{l}\text { RFM_- } \\
\text { CELL }\end{array}$ & $\begin{array}{l}\text { PROD_ } \\
\text { TYP }\end{array}$ & AGE_Y & $\begin{array}{l}\text { LSTORD_ } \\
\mathrm{M}\end{array}$ & $\begin{array}{l}\mathrm{NEW}_{-} \\
\text {VAR1 }\end{array}$ & $\begin{array}{l}\mathrm{NEW}_{-} \\
\text {VAR4 }\end{array}$ & $\begin{array}{l}\text { NEW_ } \\
\text { VAR5 }\end{array}$ \\
\hline FS & & & $\mathrm{x}$ & & $\mathrm{x}$ & & $\mathrm{x}$ & $\mathrm{x}$ \\
\hline $\mathrm{BE}$ & $\mathrm{x}$ & & & $\mathrm{x}$ & $\mathrm{x}$ & & & $\mathrm{x}$ \\
\hline SW & & & $\mathrm{x}$ & & $\mathrm{x}$ & & $\mathrm{x}$ & $\mathrm{x}$ \\
\hline$R$-sq & & & $\mathrm{x}$ & & $\mathrm{x}$ & $\mathrm{x}$ & & $\mathrm{x}$ \\
\hline Top-4 & & $\mathrm{x}$ & & & & $\mathrm{x}$ & $\mathrm{x}$ & $\mathrm{x}$ \\
\hline
\end{tabular}

fall between the second and third best original variables, RFM_CELL and PROD_TYP

- NEW_VAR2 and NEW_VAR3 are ranked eleventh and twelfth in importance before the last two significant original predictors

- NEW_VAR6 barely meets significance with a $0.0438 \mathrm{p}$-value.

\section{GMAX variable selection}

The GMAX-constructed variables plus the GMAX-selected variables can be thought of as an enhanced best subset that reflects an honest determination of necessary variables with respect to the criterion of maximising the deciles. For the illustration data, the enhanced set consists of 15 variables: DOLLAR_2, RFM_CELL, PROD_TYP, AGE_Y, LSTORD_M, and NEW_VAR1 through NEW_VAR10. The predictive power of the enhanced set is assessed by comparing logistic regression models with the geneticbased best subset, and then with the statistics-based best subset.

Using the enhanced set, five logistic regression analyses corresponding to the five variable selection methods were performed. The resultant genetic-based best subsets are displayed in Table 9. The forward, backward and stepwise methods produced different subsets equal to size 4. $R$-sq(4) and Top-4 also produced different subsets. It appears that NEW_VAR5 is the most important variable, as it is selected by all five methods. LSTORD_M is of second importance, as it is selected by four of the five methods. RFM_CELL and AGE_Y are the least important, as they are selected by only one method.

To assess the gains in predictive power of the genetic-based best subset over the statistics-based best subset, an 'average' measure of model performance for the five methods was defined based on the enhanced set: AVG-g, the average of the cum lifts across the five methods for each decile. Comparison of AVG-g with AVG 
Table 10

\section{MODEL PERFORMANCE COMPARISON BASED ON THE GENETIC-BASED BEST SUBSETS: CUM LIFT}

\begin{tabular}{lcccccccc}
\hline Decile & FS & BE & SW & $R$-sq & Top-4 & AVG-g & AVG & Gain \\
top & 265 & 260 & 262 & 265 & 267 & 264 & 252 & $4.8 \%$ \\
2 & 206 & 204 & 204 & 206 & 204 & 205 & 202 & $1.2 \%$ \\
3 & 180 & 180 & 180 & 178 & 180 & 180 & 174 & $3.0 \%$ \\
4 & 166 & 167 & 167 & 163 & 166 & 166 & 156 & $6.4 \%$ \\
5 & 148 & 149 & 149 & 146 & 149 & 148 & 144 & $3.1 \%$ \\
6 & 135 & 137 & 137 & 134 & 136 & 136 & 131 & $3.3 \%$ \\
7 & 124 & 125 & 125 & 123 & 125 & 124 & 123 & $1.0 \%$ \\
8 & 116 & 117 & 117 & 116 & 117 & 117 & 114 & $1.9 \%$ \\
9 & 108 & 108 & 108 & 107 & 108 & 108 & 107 & $0.7 \%$ \\
bottom & 100 & 100 & 100 & 100 & 100 & 100 & 100 & $0.0 \%$ \\
\hline
\end{tabular}

(average model performance based on the statistics-based set) indicates significant gains in predictive power obtained by the GMAX variable selection technique. The percentage gains range from an impressive 6.4 per cent (at the sixth decile) to a slight 0.7 per cent (at the ninth decile). The mean percentage gain for the most actionable depth-of-file, the top four deciles, is 3.9 per cent. (See Table 10.)

This illustration demonstrates the power of the GMAX variable selection technique over the current statisticsbased variable selection methods. GMAX variable selection is a high-performance method for direct marketing models with data mining capability. This method is significant in that it locates the best subset of variables to maximise the cum lift criterion.

\section{NON-LINEAR ALTERNATIVE TO} LOGISTIC REGRESSION MODEL

The GMAX model itself offers a non-linear alternative to the logistic regression model (LRM). LRM is a linear approximation of a potentially non-linear response function, which is typically noisy, multimodal and discontinuous. LRM together with the GMAX-enhanced best subset of variables (some of which are likely to be non-linear) provide an unbeatable combination of traditional statistics improved by new machine learning intelligence. This hybrid GMAXLRM model is still, however, a linear approximation of a potentially non- 
Table 11

MODEL PERFORMANCE COMPARISON LRM AND

GMAX MODEL: CUM LIFTS

\begin{tabular}{llllll}
\hline & & & & \multicolumn{2}{c}{ GMAX gain over } \\
\cline { 5 - 6 } Decile & AVG-g (Hybrid) & AVG (LRM) & GMAX & Hybrid & LRM \\
& & & & & \\
top & 264 & 252 & 283 & $7.1 \%$ & $12.2 \%$ \\
2 & 205 & 202 & 214 & $4.4 \%$ & $5.6 \%$ \\
3 & 180 & 174 & 187 & $3.9 \%$ & $7.0 \%$ \\
4 & 166 & 156 & 171 & $2.9 \%$ & $9.5 \%$ \\
5 & 148 & 144 & 152 & $2.8 \%$ & $5.9 \%$ \\
6 & 136 & 131 & 139 & $2.5 \%$ & $5.9 \%$ \\
7 & 124 & 123 & 127 & $2.2 \%$ & $3.2 \%$ \\
8 & 117 & 114 & 118 & $1.3 \%$ & $3.3 \%$ \\
9 & 108 & 107 & 109 & $1.2 \%$ & $1.9 \%$ \\
bottom & 100 & 100 & 100 & $0.0 \%$ & $0.0 \%$ \\
\hline
\end{tabular}

linear response function. Consequently, the non-linear GMAX model offers further improvement over the hybrid GMAX-LRM (and of course over LRM).

The GMAX model, as defined by the entire tree with all its structure, offers potentially superior results. Because the degree of non-linearity in the response function is never known, the best approach when modelling response is to compare the GMAX model and the hybrid GMAX-LRM model. If the improvement is determined to be stable and significant, then the GMAX model should be used.

The illustration demonstrates how the GMAX model produces cum lifts as indicated in Table 11. The GMAX model offers significant improvement over the performance of the hybrid GMAX-LRM model (AVG-g). The percentage gains range from an impressive 7.1 per cent (at the sixth decile) to a respectable 1.2 per cent (at the ninth decile). The mean percentage gain for the most actionable depth-of-file, the top four deciles, is 4.6 per cent.

The improvement of the GMAX model over the performance of the LRM (AVG) has been included. The mean percentage gain for the most actionable depth-of-file, the top four deciles, is 8.6 per cent which includes a huge 12.2 per cent in the top decile. (See Table 11.)

There are four GMAX models that produced reported performance gains. 
Figure 19: Best GMAX model



Figure 20: Best GMAX model

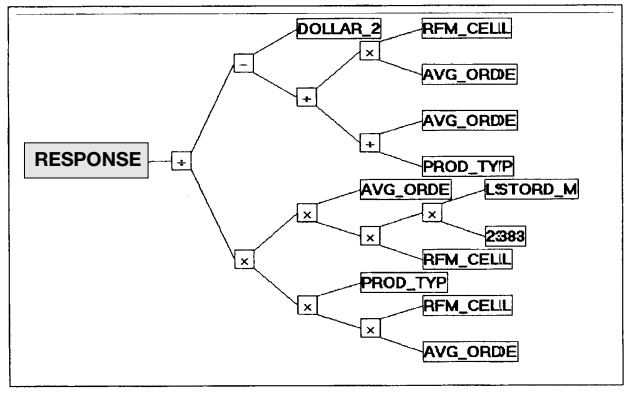

Figure 21: Best GMAX model

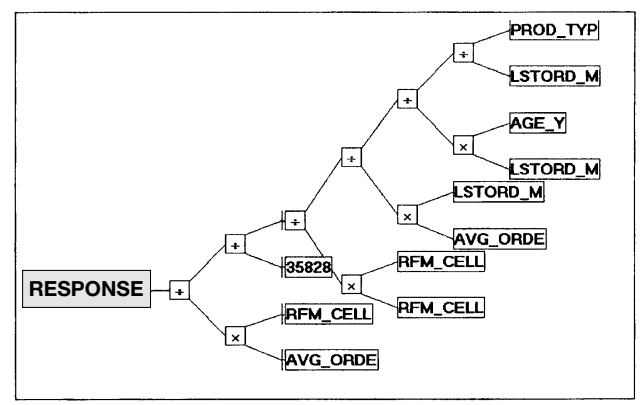

Figure 22: Best GMAX model

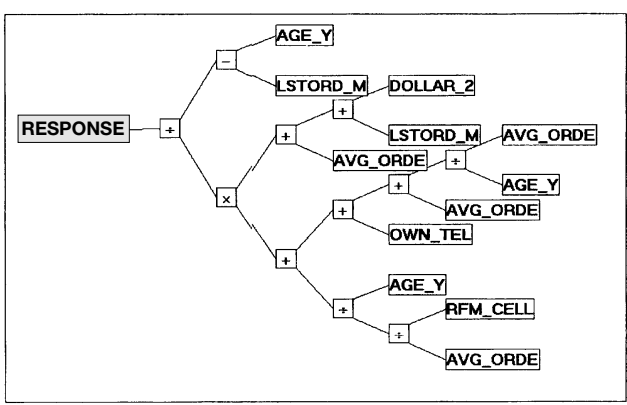


The GMAX model that produced the top, second and third deciles are in Figures 18a, 19, 20 and 21. The GMAX model that produced the fourth to bottom deciles is in Figure 21. The four separate GMAX models were needed to obtain the reported decile-performance levels because no single GMAX model could be evolved to provide gains for all upper deciles.

A set of GMAX models is required when the response function is nonlinear with noise, multi-peaks and discontinuities. The capability of GMAX to generate many models with desired performance gains reflects the flexibility of the GMAX paradigm. It allows for intelligent and adaptive modelling of the data to account for the variation of an apparent non-linear response function.

This illustration shows the power of the GMAX model as a non-linear alternative to the logistic regression model. GMAX provides a two-step procedure for response modelling. First, build the best hybrid GMAX-LRM model. Secondly, select the best GMAX model. If the GMAX model offers a stable and significant improvement over the hybrid model, then the GMAX model is the preferred response model.

As previously mentioned, the GMAX model works equally well for finding structure in a profit model. Accordingly, GMAX is a non-linear alternative to the ordinary least-squares regression model (OLS). The GMAX model offers a potentially stable and significant improvement over OLS and the hybrid GMAX-OLS model.

\section{CONCLUSION}

After reviewing the problem of variable selection and illustrating the popular statistics-based solutions, the author pointed out two common weaknesses of the popular variable selection methods: they neither identify structure, nor do their selection criteria explicitly address the specific needs of direct marketing models. He presented the GMAX model instead as a genetic-based approach for variable selection for direct marketing models. The GMAX model was demonstrated as a high-performance variable selection method with data mining capability for finding important structure to maximise the cum lift criterion. Lastly, the GMAX model itself was presented as a non-linear alternative to the linear logistic and ordinary regression models, most useful when it is assumed the response and profit functions are non-linear with noise, multi-peaks and discontinuities.

\section{REFERENCES}

1 Other criteria are based on information theory, and Bayesian rules.

2 Dash, M. and Liu, H. (1997) 'Feature selection for classification', Intelligent Data Analysis, Elsevier Science Inc.

3 SAS/STAT Manual. See PROC REG, and PROC LOGISTIC.

$4 R$-squared theoretically is not the appropriate measure for a binary dependent variable. However, many analysts use it with varying degrees of success.

5 For example, consider two TS values: 1.934056 and 1.934069. These values are equal when rounding occurs at the 
third place after the decimal point: 1.934.

6 Absolute $r$ value means that the sign is ignored. Eg if $r=-0.23$ then absolute $r=+0.23$.

7 Ryan, T. P. (1997) 'Modern regression methods', Wiley.

8 Miller, A. J. (1990) 'Subset selection in regression', Chapman and Hall.

9 The weights or coefficients $\left(b_{0}, b_{1}, b_{2}\right.$ and $b_{3}$ ) are derived to satisfy some criterion, such as minimise the mean squared error used in ordinary leastsquare regression, or minimise the joint probability function used in logistic regression.

10 Fox, J. (1997) 'Applied regression analysis, linear models, and related methods', Sage Publications.

11 The term 'profit' is used as a standin for any measure of an individual's worth, such as sales per order, lifetime sales, revenue, number of visits or number of purchases.

12 The focus of this paper is optimisation; but genetic modelling has been applied to a variety of problems: optimal control, planning, sequence induction, empirical discovery and forecasting, symbolic integration and discovering mathematical identities.

13 Genetic modelling as described in this paper is formally known as genetic programming, developed by John Koza, 'Genetic programming: On the programming of computers by means of natural selection', The MIT Press, Cambridge, MA.

14 The term 'modelling' is used instead of 'programming' because the latter term, which has its roots in computer sciences, does not connote the activity of model building to data analysts with statistics or quantitative backgrounds.

15 Effectively, a genetic alphabet has been chosen.

$16 R$-squared is not an appropriate fitness function for a $0-1$ dependent variable model. Perhaps the likelihood function of the logistic regression model should have been used as the fitness measure for the response model, or an example with a continuous (profit) variable and the $R$-squared fitness measure. There is more about the appropriate choice of fitness function for the problem at hand in a later section.

17 When a subtree has more than two terminals the terminals are randomly permutated.

18 RFM_CELL will be treated as a scalar variable.

19 The direction of the association is not relevant. That is, the sign of the coefficient is ignored.

20 The log to the base 10 also symmetrises dollar amount variables.

21 Top fitness values typically bunch together with equivalent values. The top fitness values are considered equivalent in that their values are equal when rounded at, say, the third place after the decimal point. Consider two fitness value: 1.934056 and 1.934069 . These values are equal when rounding occurs at, say, the third place after the decimal point: 1.934 .

22 The mean incidence cut-off score of 0.75 has been empirically pre-determined. 